\title{
ZWISCHEN NEAPEL UND MADRID: VICENTE MARTÍN Y SOLER UND DAS SPANISCHE KÖNIGSHAUS
}

\author{
Rainer Leonhard KLEINERTZ \\ Universität Regensburg
}

Der 1754 in Valencia geborene Vicente Martín y Soler ist wohl der einzige spanische Komponist des 18. Jahrhunderts, der weit über die Grenzen seines Vaterlands hinaus bekannt war. Seine Berühmtheit —zumeist als «Martini lo Spagnuolo»- verdankte er allerdings keinem spanischen Werk, sondern ausschließlich italienischen Opern, allen voran dem 1786 in Wien uraufgeführten Dramma giocoso Una cosa rara o sia Bellezza ed onestà auf einen Text von Lorenzo Da Ponte ${ }^{1}$. Dabei dürfte der durchschlagende Erfolg dieses Stückes, das in Wien eine wahre Cosa-rara-Mode entfachte ${ }^{2}$, nicht zuletzt auch auf das spanische Ambiente der Handlung zurückzuführen sein, dem durch die Vertonung eines Spaniers der Anschein von Authentizität verliehen wurde ${ }^{3}$. Es entbehrt allerdings nicht einer gewissen Ironie, daß Martín y Soler, dessen Cosa rara immerhin Mozarts kurz zuvor uraufgeführte Le nozze di Figaro vom Spielplan verdrängte, in unserem Jahrhundert fast nur mehr durch das Zitat aus der Cosa rara im zweiten Finale von Mozarts Don Giovanni bekannt ist ${ }^{4}$.

Martín y Solers Werdegang als Komponist liegt jedoch bis heute weitgehend im Dunkeln ${ }^{5}$. Nach seiner Kindheit in Valencia, wo er an der Iglesia Mayor, an der sein Vater Tenorist

1. Eine kritische Ausgabe dieses Werkes von Gerhard Allroggen erschien 1990 im Henle-Verlag, München (Die Oper. Kritische Ausgabe von Hauptwerken der Operngeschichte, hrsg. von Heinz Becker, Bd. 5).

2. Vgl. die Einleitung von Allroggen zu seiner Edition der Oper, S. VI.

3. Die Handlung spielt in einem Ort in der Sierra Morena. Dabei griff Da Ponte zurück auf La luna della sierra von Luis Velez de Guevara, das er fälschlich für ein Werk Calderóns hielt. Die Bedeutung des Ambientes für den Erfolg des Stückes unterstreicht auch ein Bericht des Holsteinischen Correspondenten vom 23. Januar 1787: „Die von dem Spanischen Kapellmeister Martini zu Wien in Musik gesetzte neue Oper: La cosa rara, erhält in Wien wegen des darinn herrschenden Spanischen Geschmacks ganz außerordentlichen Beyfall" (zitiert nach Allroggen, S. VII). Musikalisch reflektiert sich dieser stilisierte ,Folklorismus' beispielsweise in der Seghidiglia des zweiten Finales. Vgl. den entsprechenden Artikel von Silke LeOPold in Pipers Enzyklopädie des Musiktheaters, hrsg. von Carl Dahlhaus u. a., Bd. 3, München u. Zürich, 1989 , S. 703 f.

4. Zitiert wird in der Tafelmusik der Schlußsatz des ersten Finales der Cosa rara, «O quanto un sì bel giubilo». Das Zitat ist bereits im Libretto vorgesehen, das ebenso wie das der Cosa rara von Da Ponte stammt.

5. Vgl. die Einleitung von Dorothea Eva Link zu ihrer Dissertation über The Da Ponte Operas of Vicente Martín y Soler, Diss. University of Toronto 1991, S. $4 \mathrm{ff}$ 
war, zum Chorknaben ausgebildet wurde ${ }^{6}$, ging Martín y Soler angeblich nach Madrid, wo er auf Anregung des Baritons Domenico Guglietti einige Arien komponiert haben soll ${ }^{7}$. Allerdings gibt es bislang weder in den Akten des Hofes (einschließlich der Capilla Real) noch in den erhaltenen Theaterdokumenten im Stadtarchiv irgendeinen Hinweis auf seine Tätigkeit in Madrid. Als sein erster Versuch auf der Opernbühne gilt allgemein eine «Zarzuela» mit dem Titel La Madrileña, deren Uraufführung man bislang —zumeist mit einem Fragezeichen versehen- für 1776 in Madrid annahm.

Das erste gesicherte Datum seines Lebens - mit Ausnahme seines Geburtsdatums (und selbst das war lange Zeit ungewiß) - ist eine Aktennotiz im Staatsarchiv von Neapel vom 17. Juli 1778, daß ein Gläubiger des «D. Vincenzo Martin, Maestro di Cappella Spagnuolo, addetto al R. Teatro di S. Carlo» den König um Beistand bei der Eintreibung seiner Schulden bitte ${ }^{8}$. Ulisse Prota-Giurleo führt an, daß bereits am 20. Januar desselben Jahres im Teatro di S. Carlo die Musik Martín y Solers zu dem Ballett Li novelli sposi persiani von Charles Lepicq aufgeführt worden war ${ }^{9}$. Dieser «Ballo» bildete den ersten Zwischenakt des Dramma per musica Bellerofonte von Ignazio Platania, das zur Feier des Geburtstags des spanischen Königs Carlos III. ${ }^{10}$ aufgeführt wurde und seinem Sohn und König von Neapel Ferdinando IV. gewidmet war ${ }^{11}$. Man wird also davon ausgehen dürfen, daß Martín y Soler sich bereits seit Ende 1777 in Neapel aufhielt.

6. Vgl. die Artikel "Martín y Soler" von Othmar Wessely in Die Musik in Geschichte und Gegenwart (MGG), hrsg. von Friedrich Blume, Bd. 8, Kassel u. a. 1960, Sp. 1710 ff. und The New Grove Dictionary of Music and Musicians, hrsg. von Stanley Sadie, London 1980, Bd. 11, S. 735 f., sowie von Dorothea Link in The New Grove Dictionary of Opera, hrsg. von Stanley Sadie, London 1992, Bd. 3, S. 240 f.

7. Eine Erklärung für die oft wiederholte Behauptung, Martín y Soler sei Organist an einer Kirche in Alicante gewesen, wofür sich keinerlei Belege finden ließen, gibt ein bisher unbeachteter biographischer Artikel über Martín y Soler im ersten Jahrgang der Zeitschrift La Iberia musical (1842, Nr. 6, S. 21f.: Estudios biográficos. Artistas célebres españoles. Vicente Martín). Darin heißt es, Martín habe sich, nachdem er Chorknabe an der Kathedrale von Valencia gewesen war, zwar um einen Platz als Organist an einer Kirche in Alicante beworben, habe die Stelle jedoch nicht bekommen. Daraufhin sei er nach Madrid gegangen, wo er Freundschaft mit dem neapolitanischen Sänger Guglietti geschlossen und für diesen mehrere Arien geschrieben habe.

8. Das Dokument ist zitiert bei Ulisse Prota-Giurleo, «Del compositore spagnuolo Vincente Martin y Soler», Archivi 27 (1960), S. 145-156, hier S. 145: «Onofrio di Jorio, trattor pubblico, essendo creditore, per somma di duc. 7 e rotti, di D. Vincenzo Martin, Maestro di Cappella Spagnuolo, addetto al R. Teatro di S. Carlo, e ciò per vitto somministratogli, implora gl'ordini Sovrani, non potendo esser soddisfatto per altra via» (Teatri, fasc. 22).

9. «Noi, peraltro, siamo riusciti ad accertare che fin dal novembre 1777 il Martin era stato assunto dal famoso ballerino e coreografo Charles Lepicq per mettere in musica il Ballo, che questi andava componendo per il teatro S. Carlo. La spettacolosa azione coreografica, che andò in iscena il 20 gennaio 1778 insieme col Bellerofonte del palermitano Ignazio Platania, s'intitolava La Sposa persiana, e riportò un successo strepitosissimo» (ibid., S. 146). Leider gibt Prota-Giurleo weder für die Autorschaft Martín y Solers an der genannten Ballettmusik noch für deren durchschlagenden Erfolg Quellen an.

10. Carlos III. überließ 1759, als er seinem Bruder Fernando VI. auf den spanischen Thron folgte, die Krone beider Sizilien seinem zweitältesten Sohn Ferdinand, der als Ferdinand IV. König von Neapel und als Ferdinand I. König beider Sizilien wurde.

11 «Bellerofonte. Dramma per musica da rappresentarsi nel Real Teatro di S. Carlo nel dì 10 gennaro 1778 in cui si festeggia la nascita della S. R. C. M. di Carlo III monarca delle Spagne ed alla S. R. M. di Ferdinando IV suo amabilissimo figlio e nostro clementissimo re dedicato. Napoli, Vincenzo Flauto, 1778» (zitiert nach Claudio SARToRI, I libretti italiani a stampa dalle origini al 1800, 7 Bde., Cuneo 1990-1994, Nr. 3935). Der zweite Ballo war Gli amanti protetti dalla magia von Domenico Rossi. Die Komponisten der Balli (sofern Martín y Soler nicht die Musik zu beiden Balli verfaßte) sind im Libretto nicht angegeben. 
Das Ansehen, das er in Neapel bereits 1778 zumindest bei Hofe genoß, läßt ein Konzert am 21. Juli 1778 vor dem Palast von Mergellina erkennen, an dem Martín y Soler und der König gemeinsam mitwirkten, wie die Gazzetta Universale berichtet:

«21 luglio 1778 - Ieri sera nella Piazza che rimane davanti al Real soggiorno di Mergellina, furono situati 20 piccoli cannoni, al fianco dei quali era un'Orchestra di Musica, formata di 40 violini, molti controbassi e strumenti da fiato. Un Maestro di Cappella Spagnuolo, inventore di questo Concerto, portava la battuta. Il Re e il Principe di Butera erano gli Artiglieri con le micce in mano. Si suonò dunque una strepitosa sinfonia, e quando dovevano i cannoni sparare in cadenza, gridava il Maestro $\langle F u e g o !>$, e si faceva questo ora con due, ora con quattro ora con sei tiri, secondo il concerto. Tale spettacolo richiamò una folla di popolo immensa, e fu ammirata l'affabilità dei Sovrani, tutti addetti al divertimento dei sudditi». ${ }^{12}$

Am folgenden Tag erging der königliche Befehl, daß die Oper für den nächsten Karneval von Martín y Soler komponiert werden solle ${ }^{13}$. Tasächlich ging am 12. Januar 1779, dem Geburtstag Ferdinands, im Teatro di S. Carlo das Dramma per musica von Luigi Serio Ifigenia in Aulide mit der Musik von Vicente Martín y Soler in Szene ${ }^{14}$.

Vor dem Hintergrund dieser wenigen Informationen kommt dem in der Biblioteca Histórica Municipal de Madrid aufbewahrten Aufführungsmaterial zu La Madrileña über die Tatsache hinaus, daß es die einzige erhaltene Quelle für dieses Werk ist, ein besonderer dokumentarischer Wert zu. Erhalten sind dort eine Partitur mit Stimmensatz sowie drei handschriftliche Libretti mit einem «Guión de música» (ein Textbuch, das nur die musikalischen Teile enthält) ${ }^{15}$. Das Titelblatt der Partitur lautet: «Il tutore Burlato/ Musica Del Sig. ${ }^{r}$ Vincezo [sic] Martin/ Detto Il Valenziano/ Nel Real Sito Di S. Ildifonso/ anno 1778». Das Werk wurde also allem Anschein nach 1778 im Theater oder einem anderen geeigneten Platz des königlichen Palastes von La Granja in San Ildefonso (bei Segovia) aufgeführt oder sollte zumindest dort aufgeführt werden. Wer erwartet hatte, hier nun den ,unverfälschten ' spanischen Martín y Soler kennenzulernen, eine Zarzuela von einem Komponisten, der auch über die iberische Halbinsel hinaus bekannt ist, wird allerdings enttäuscht: Tatsächlich handelt es sich um eine dreiaktige italienische Opera buffa, die erst nachträglich ins Kastilische übersetzt wurde. So enthält die Partitur nicht allein alle musikalischen Nummern mit italienischem Text, sondern auch die (Secco-) Rezitative. In den Musiknummern wurde anschließend zusätzlich der kastilische Text unterlegt, während dies bei den Rezitativen, deren Text bei einer Aufführung in spanischer Sprache natürlich gesprochen wurde, unterblieb. Das Ausschreiben der Rezitative in der Partitur war also nur sinnvoll, wenn das Werk zunächst für eine Aufführung in italienischer Sprache vorgesehen war.

Auf der ersten Seite des ersten Aktes («Atto Primo / Introduzione») ist oben links vermerkt: «ㄹ [=Duetto] Zarz. ${ }^{a}$ de [?] La Madrileña, ò tuttor Burlado.» Die Handschrift ist offen-

12. Zitiert nach Prota-Giurleo, Del compositore spagnuolo Vincente Martin y Soler, S. 146.

13. Ibidem.

14. Vgl. SARTORI, I libretti italiani a stampa, Nr. 12729.

15. Die Partitur und die Stimmen haben die Signatur Mus 57-14, die Libretti die Signatur Tea 189-6. 
sichtlich dieselbe wie die der Eintragung des spanischen Textes unter dem Italienischen, es handelt sich also um einen Hinweis darauf, daß dasselbe Werk in kastilischer Sprache unter dem Titel La Madrileña aufgeführt wurde. Dies entspricht auch dem Titel der handschriftlichen spanischen Textbücher: «Zarzuela/ La Madrileña./ ò el/ Tutor burlado./ Para la comp./ de/ Manuel Martinez.» Das dritte Exemplar enthält Zensurvermerke und die Approbation vom 20. Juli $1778^{16}$. Ein gedrucktes Libretto konnte bisher nicht nachgewiesen werden.

Merkwürdigerweise gibt es über das Aufführungsmaterial hinaus bislang überhaupt keine Hinweise auf irgendeine Aufführung des Werkes. Die Approbation legt jedoch nahe, daß zumindest eine spanischsprachige Aufführung als La Madrileña tatsächlich stattgefunden hat. Zwar stehen weder das genaue Datum noch die Zahl der Aufführungen fest, doch wird man die Erstaufführung für die Tage nach dem 20. Juli vermuten dürfen. Wie das Titelblatt des Librettos erkennen läßt, waren die Ausführenden Mitglieder der Gesellschaft («compañia») von Manuel Martínez, die neben der von Eusebio Ribera die Madrider Theater de la Cruz und del Príncipe bespielte und der neben Schauspielern auch Sänger angehörten. So verzeichnet die Liste der Mitglieder, die im März 1778 für dieses Jahr aufgestellt wurde ${ }^{17}$, eine 〈tercera dama de cantar〉, María Antonia Fernández alias La Caramba ${ }^{18}$, «con la sola obligación de cantar», was auch für die vierte Dame, Nicolasa Palomera, galt, sowie ferner einen 〈primer gracioso〉, Miguel Garrido, «con obligación de cantar solamente en los Teatros y funciones de Musica». Hinzu kam als «Musico» Manuel Ferreyra, neben den im darauffolgenden Jahr mit Pablo Esteve noch ein «Compositor» trat. Wenn es sich also auch nicht um eine Operngesellschaft handelte, so war die Aufführung von musikalischen Werken doch durchaus vorgesehen und ständig geübte Praxis. (Daß hierzu gerade auch Übersetzungen italienischer Opern gehörten, zeigt - um nur ein Beispiel von vielen zu nennen - die Aufführung der «Zarzuela» La isla de la pescadora 1778, einer spanischen Bearbeitung von Niccolò Piccinnis La pescatrice $)^{19}$.

Mit Hilfe der erwähnten Mitgliederliste der Gesellschaft von Manuel Martínez lassen sich die in Libretti, Partitur und Stimmen meist nur mit Vornamen oder abgekürzt genannten Sänger ermitteln:

Violante - enamorada de Pipo: Sra. Nicolasa Palomera

Don Lelio - caballero enamorado de Violante: Sebastian Briñoli

Dña. Menica - hija de Fabricio: Sra. Maria Antonia Fernandez, alias «la Caramba»

Fabricio - tutor de Violante: Diego Coronado [ $«$ Cor. $\left.^{\mathrm{do}}{ } »\right]$

Pipo - pastor enamorado de Violante: Miguel Garrido

Anselmo - criado de Don Lelio: Antonio Robles

16. Von Thomas Antonio Fuentes vom 7. Juli und Fr. José Puerta Palanco vom 8. Juli. Schließlich die Approbation durch den Corregidor von Madrid, José Antonio de Armona vom 20. Juli 1778.

17. Biblioteca Nacional de Madrid, Legado Barbieri, Mss. 14074 (125).

18. María Antonia Fernández, genannt «La Caramba», war eine der berühmtesten Sängerinnen von Tonadillas; vgl. Antonina Rodrigo, María Antonia La Caramba. El genio de la tonadilla en el Madrid goyesco, Madrid, Editorial «Prensa española», 1972.

19. Das Manuskript mit dem Datum 1778 befindet sich in der Biblioteca Histórica Municipal de Madrid. 
In dieser Besetzung also dürfte Martín y Solers Madrileña im Sommer 1778 im Madrider Teatro de la Cruz oder del Príncipe zur Aufführung gekommen sein.

Schwieriger ist die Frage zu beantworten, wer das Werk in San Ildefonso aufführte und wann dies geschah. In den königlichen Schlössern (Sitios Reales) fanden laut Luis Carmena y Millán nach 1776 bis 1849 keine Opernaufführungen mehr statt. 1777 habe Carlos III. dort jede Art von Aufführung verboten. Guglielmis La sposa fedele sei im Sommer 1776 die letzte Opernaufführung gewesen. In einer Fußnote merkt Carmena hierzu an:

\begin{abstract}
«Esta debe ser la última ópera italiana representada en los Reales teatros, pues á pesar de las investigaciones practicadas, no hemos hallado libreto alguno posterior á esta fecha. En el año de 1788 [!], y en el Real Sitio de Aranjuez, todavia se representó una comedia con música titulada El Barbero de Sevilla, precedida de la siguiente loa: <Los Vaqueros de Aranjuez: loa ó introduccion para la fiesta El Barbero de Sevilla, que se representó á SS. AA. en la Casa de las Vacas del Real Sitio de Aranjuez, de órden del Excelentísimo Sr. Conde de Floridablanca, primer Secretario de Estado, etc., etc., el dia 25 de Junio de este año 1788 [also noch zur Regierungszeit von Carlos III.]. Escribióla D. Ramon de la Cruz y Cano.> Tomaron parte en la loa, y probablemente en la comedia, los artistas siguientes: Antonio Robles, Vicente Camas, Sebastian Briñoli, Alfonso Navarro, Juan Antolin, Vicente Romero, José Ordoñez, Tadeo Palomino, Mariano Querol, José García Ugalde, Juan Aldobera, Manuel García Parra, Miguel Garrido e Ignacio Cadenas. No hemos podido averiguar quién fue el autor de la citada comedia.» ${ }^{20}$
\end{abstract}

Bei der erwähnten «fiesta» El barbero de Sevilla dürfte es sich mit ziemlicher Sicherheit um die bekannte Oper Paisiellos mit gesprochenen Dialogen gehandelt haben («Fiesta» wurde oft als Synonym für «Zarzuela» gebraucht), die im Dezember 1787 in spanischer Übersetzung im Madrider Teatro del Príncipe zur Aufführung gekommen war ${ }^{21}$. Es ist in diesem Zusammenhang nicht uninteressant, daß die Namen der Schauspieler bzw. Sänger teilweise mit denen der Madrileña von 1778 übereinstimmen. Es handelte sich also ebenfalls um Mitglieder Madrider Schauspielgesellschaften, die 1788 —zehn Jahre später- im Palast von Aranjuez auf Anweisung des Grafen von Floridablanca, gewissermaßen des ,Ministerpräsidenten ' Carlos' III., eine Oper zur Aufführung brachten. Bemerkenswert ist ferner, daß die Aufführung nicht etwa im Theater des Palastes stattfand, sondern in der Casa de las Vacas, einem offensichtlich für gesellige Zwecke umgestalteten Nutzgebäude ${ }^{22}$. Dies verweist auf eine neue Funktion von Opernaufführungen am Hof: Hier ging es nicht mehr um eine Selbstdarstellung des Monarchen vor seinen Untertanen, sondern um ein Divertissement der königlichen Familie selbst, bei dem nur mehr ein enger Kreis von Vertrauten erwünscht war.

20. Luis Carmena y Millán, Crónica de la ópera italiana en Madrid desde el año 1738 hasta nuestros días, Madrid 1878, S. 18.

21. Vgl. Carmena y Millán, Crónica de la ópera italiana en Madrid, S. 53. - Grundsätzlich ist aber auch nicht auszuschließen, daß es sich um das ,Originalwerk' von Beaumarchais handelte, das 1780 von Manuel Fermin de Laviano übersetzt worden war (vgl. Francisco Aguilar Piñal, Bibliografía de autores españoles del siglo XVIII, Bd. 5, Madrid, CSIC, 1989, Nr. 554).

22. Eine Ansicht dieses Gebäudes auf einem Stich von 1775 befindet sich in der Biblioteca Nacional de Madrid, Sección de Bellas Artes, Inv. 23143 (abgebildet in dem Ausstellungskatalog El Real Sitio de Aranjuez y el Arte Cortesano del siglo XVIII, Salas de exposiciones del Palacio Real de Aranjuez, abril-mayo 1987, Madrid, Comunidad de Madrid und Patrimonio Nacional, 1987, S. 373, Nr. 134). 
Das Problem für den Musikhistoriker liegt darin, daß solche nicht- oder halböffentlichen Ereignisse kaum dokumentiert sind. Hinzu kommt in diesem Falle noch die Schwierigkeit, daß die Aufführungen von Opern an den «Sitios Reales» in der Umgebung von Madrid -El Pardo, Aranjuez, El Escorial und San Ildefonso-, an denen der Hof jeweils einen Teil des Jahres verbrachte, bisher insgesamt kaum erforscht sind.

Eine Aufführung der Madrileña in San Ildefonso ist jedenfalls nur während der Anwesenheit des Hofes denkbar, der sich 1778 vom 8. August bis zum 10. Oktober dort aufhielt. Daß es in dieser Zeit dort im Theater Aufführungen gab, legt das Gesuch eines Seiltänzers («Autor de Bolatines») namens Pedro Cardona nahe, ihm den «casco del Coliseo del Real Sitio de San Ildefonso» zu überlassen, um dort Kunststücke und Tänze aufzuführen und auch einige «tonadillas a solo y acompañadas» zu singen, ferner «Arias, y conciertos á solo» ${ }^{23}$. Solche ,ephemeren' Spektakel legen jedenfalls nahe, daß auch das Theater selbst bespielt wurde und somit Zuschauer anzog.

$\mathrm{Ob}$ in diesen Wochen neben der königlichen Familie vielleicht auch die Königin von Neapel, Maria Carolina (1752-1814), anwesend war, eine Schwester Kaiser Josephs II., die 1768 Ferdinand IV. von Neapel geheiratet hatte ${ }^{24}$, ist nicht belegt. Jedenfalls wurde am 13. August ihr Geburtstag feierlich begangen, wie die Gazeta de Madrid am 18. August 1778 meldete:

«En celebridad del feliz cumpleaños de la Reyna de Nápoles se vistió el 13 del corriente la Corte de gala, y hubo besamanos general en el Real Sitio de S. Ildefonso con el mas lucido concurso de Grandes, Diputados de los Reynos, Embaxadores y Ministros estrangeros, y otras personas de distincion. ${ }^{25}$

Ein Aufenthalt der Königin von Neapel in San Ildefonso legt aber zugleich nahe, daß auch ihr Gemahl, König Ferdinand, — der Sohn Carlos' III. - anwesend war. Beide waren große Theaterliebhaber, wie ein Bericht des Sängers Michael Kelly über den neapolitanischen Hof in seinen Reminiscences erkennen läßt:

"The King was very tall, near-sighted, with very light eye-brows, and remarkably fair hair. He was very partial to theatricals, and when he went to Caserta (one of his palaces), in which there was a beautiful little theatre, he often condescended to act in burlettas ${ }^{26}$ with the Queen. They uniformly took the parts of the principal Buffo and Buffa; the graver and more important characters were filled by the Lords and Ladies of the Court." 27

23. Archivo del Palacio Real de Madrid, Carlos III, Reales Ordenes, Años 1773-1781, Leg. 9. Dem Gesuch vom 19. Juni 1778 aus Aranjuez wurde am 26. Juni positiv entsprochen.

24. Seit 1777 führte sie weitgehend die Regierungsgeschäfte. $\mathrm{Zu}$ den Beziehungen zwischen dem Madrider und dem neapolitanischen Hof, die sich nach 1778 deutlich verschlechterten, vgl. den Beitrag von Rafael OlaECHEA, «Relaciones diplomáticas entre España y el reino de Nápoles a fines del '700», in: I Borbone di Napoli e $i$ Borbone di Spagna. Un bilancio storiografico, hrsg. von Mario Di Pinto, Napoli, Guida editori, 1985 (Acta Neapolitana 3), Bd. 2, S. 7 ff.

25. Gazeta de Madrid, Nr. 33 vom 18.8.1778, S. 335

26. Mit "burlettas" dürften hier - dem englischen Sprachgebrauch der Zeit entsprechend- kleinere komische Opern, insbesondere Intermezzi gemeint sein. Vgl. den Artikel "Burletta" in The New Grove Dictionary of Music and Musicians, Bd. 3, S. $473 \mathrm{f}$.

27. Michael Kelly, Reminiscences, 2 Bde., London 1826, Reprint mit einer Einleitung von A. Hyatt King: New York 1968, Bd. 1, S. 51. - Die Sitte, in kleineren Opern mitzuwirken, war Maria Carolina bereits aus Wien vertraut, wo mehrere kleinere Werke Metastasios - anscheinend alle pastoralen Charakters - von Mitgliedern der kaiserlichen Familie und des Hofes aufgeführt wurden. Vgl. den Artikel "Metastasio" in The New Grove Dictionary of Music and Musicians, Bd. 
Bedenkt man, daß Martín y Soler sich seit 1777 in Neapel aufhielt und dort im Juni 1778 jene erwähnte ,Kanonen-Symphonie“ unter persönlicher Mitwirkung des Königs aufgeführt hatte, so wäre auch eine Anwesenheit Martín y Solers im Gefolge der Königin oder des Königs von Neapel denkbar. Dies würde immerhin erklären, warum gerade eine Oper eines damals noch unbekannten vierundzwanzigjährigen Komponisten aus Neapel, der bis dahin keine nachweisbaren Spuren in Madrid hinterlassen hatte, aufgeführt werden sollte. Betont werden muß jedoch, daß all dieses reine Spekulation ist. Sicher ist nur, daß der spanische Hof am 13. August 1778 wie schon in den Jahren zuvor den Geburtstag der Königin von Neapel feierte und daß im Jahr 1778 - und aller Wahrscheinlichkeit nach während der Anwesenheit des Hofes zwischen dem 8. August und dem 10. Oktober- eine Aufführung von Martín y Solers Oper stattfand oder zumindest beabsichtigt war.

Dabei erscheinen die folgenden Aufführungsmöglichkeiten denkbar:

1.) Mitglieder der Gesellschaft von Manuel Martínez führten das Werk, das sie zuvor schon in Madrid gegeben hatten, im Theater des Palasts von La Granja auf, entweder als einmalige ,Galavorstellung ' oder im Rahmen einer längeren ,Stagione“ als öffentliche Vorstellung ${ }^{28}$.

2.) Die Oper wurde, wie es zumindest bis 1777 an den Theatern der königlichen Paläste üblich gewesen war, von einer Gesellschaft von italienischen Sängern aufgeführt, die auch in Städten wie Valencia und Sevilla auftraten ${ }^{29}$.

3.) Die Oper wurde von Angehörigen des Hofes, vielleicht unter Mitwirkung der Königin und des Königs von Neapel — sofern sie tatsächlich anwesend waren-, im Stil der von Kelly für Neapel bezeugten Divertissements aufgeführt. (Hierzu würden auch die vergleichsweise mäßigen gesanglichen Anforderungen in den Partien der «primi buffi» Violante und Pippo mit ihrem überwiegend volkstümlich-pastoralen Ton passen $)^{30}$.

12, S. 215: "During the 1740 s and 1750 s the poet wrote several texts to be acted and sung by her [Maria Theresia's] daughters in private; and during the 1760 s and early 1770 s he completed some further librettos to honour important family occasions such as the marriages of her children or the birth of a grandchild." Maestro di musica der Erzherzoginnen Maria Carolina und Marie Antoinette war im übrigen zwischen 1760 und 1762 Johann Adolph Hasse (vgl. MGG 5, Sp. 1775).

28. Am 8. September 1778, also während des Aufenthaltes in San Ildefonso, ließ der König einem Manuel Martínez 300 Reales de vellón zukommen: «El Rey a concedido á Manuel Martinez que ha presentado el adjunto Memorial trecientos rs. de limosna por una vez» (Archivo del Palacio Real de Madrid, Carlos III, Reales Ordenes, Años 1773-1781, Leg. 9). Aufgrund der Häufigkeit dieses Namens ist jedoch unklar, ob derselbe Manuel Martínez gemeint ist. Das erwähnte Memorial, aus dem die Gründe für das Bittgesuch hervorgingen, ist leider nicht erhalten.

29. So wurde beispielsweise 1776 in San Ildefonso das Dramma giocoso Le gemelle mit der Musik von Antonio Tozzi von einer italienischen Operngesellschaft aufgeführt, der Tozzi als Kapellmeister vorstand (vgl. SARTORI, I libretti italiani a stampa, Nr. 11487, und Emilio Cotarelo Y Mori, Orígenes y establecimiento de la ópera en España hasta 1800 , Madrid 1917, S. 207). - Unter den wenigen Dokumenten zum Theater von San Ildefonso im Archiv des Palacio Real de Madrid befindet sich eine Eingabe eines «Impresario del Teatro del Real Sitio» von 1784 mit der Bitte, daß ihm die Miete wegen der geringen Einnahmen erlassen werde. Die Tatsache, daß die Comapañía der Reales Sitios 1777 aufgelöst wurde, besagt also nicht, daß es fortan in den Theatern der königlichen Paläste keine Vorstellungen mehr gegeben hätte. Ob dies spanische oder italienische Gesellschaften waren, ist jedoch unbekannt. - Vgl. in diesem Zusammenhang auch den materialreichen Aufsatz von Xoán M. CARreira, «El teatro de ópera en la Península Ibérica ca. 1750-1775. Nicolà Setaro», in: De musica hispana et aliis, Festschrift José López-Calo zum 65. Geburtstag, hrsg. von Emilio Casares u. Carlos Villanueva, Santiago de Compostela, Universidad, 1990, Bd. 2, S. $27 \mathrm{ff}$.

30. Grundsätzlich wäre auch eine frühere Aufführung in Neapel in der Art wie sie Kelly beschreibt nicht auszuschließen. 
Welche der drei Möglichkeiten der Wirklichkeit entspricht, läßt sich derzeit nicht entscheiden. Für die erste Möglichkeit spricht die Tatsache, daß die Oper offensichtlich zuvor schon in Madrid aufgeführt worden war. Andererseits bleibt in diesem Falle die Frage, warum Martín y Soler das Werk zunächst in Italienisch mit ausgeschriebenen Rezitativen komponierte und warum das Titelblatt derselben (Gebrauchs-) Partitur, in der der spanische Text nachträglich unterlegt wurde, ausdrücklich eine Aufführung in San Ildefonso 1778 vorsieht.

Schließlich ist es sogar möglich, daß die Oper von Martín y Soler zwar für San Ildefonso geschrieben bzw. kopiert wurde, dort jedoch gar nicht zur Aufführung kam. Bei allen denkbaren Möglichkeiten bleibt jedoch eine Tatsache unübersehbar: Martín y Soler war 1778 bereits in und für Neapel tätig, wo er sich am 21. Juli 1778 noch aufhielt.

Auch die Wahl des Librettos weist nach Neapel, gibt aber dennoch einige Rätsel auf. Hierbei handelt es sich um eine neapolitanische Commedia per musica von Pasquale Mililotti handelt, die 1769 in Neapel als La finta semplice ${ }^{31}$, ossia Il tutore burlato mit der Musik von Giacomo Insanguine, nach seinem Geburtsort auch Monopoli genannt, uraufgeführt worden war. Diese Oper erlebte noch zwei weitere Inszenierungen, eine 1772 in Bologna mit einigen hinzugefügten Arien von Giacomo Rust und eine weitere - die letzte nachweisbare- 1773 in Lissabon. Dabei erlebte das Libretto eine immer stärkere ,Toskanisierung ': Während in der ursprünglichen neapolitanischen Fassung von 1769 noch fünf von acht Personen neapolitanischen Dialekt sprechen, ist es in Bologna 1772 nur noch eine von sieben (der ,primo buffo'), und in der Lissabonner Fassung von 1773 sind schließlich alle Rollen ins Toskanische übertragen ${ }^{32}$. Wie der Textvergleich eindeutig zeigt, war es dieses Lissabonner Textbuch, das Martín y Soler vertonte.

Auffallend an dieser Wahl ist, daß Martín y Soler beziehungsweise der Auftraggeber dieses ursprünglich neapolitanische Libretto wählten, obwohl derselbe Text bereits 1774 durch den venezianischen Librettisten Filippo Livigni eine Bearbeitung erfahren hatte, die unter dem Titel La Frascatana mit der Musik von Giovanni Paisiello zu der wahrscheinlich meistgespielten Opera buffa des 18. Jahrhunderts wurde. (Dabei griff Livigni allerdings nicht auf die späteren Fassungen, sondern auf das neapolitanische Libretto von 1769 zurück.) Neben einer Vielzahl von Aufführungen in ganz Europa war Paisiellos Frascatana bereits 1777 in Valencia und Sevilla aufgeführt worden ${ }^{33}$, und auch der Titel der spanischen Übersetzung, La Madrileña, läßt erkennen, daß diese Oper auch in Madrid, wo die erste Aufführung erst 1782 belegt ist ${ }^{34}$, bereits bekannt war. Die Wahl der älteren Libretto-Fassung läßt sich nur so erklären, daß Martín y Soler

31. Trotz des identischen Titels hat die Handlung nichts mit dem Dramma giocoso La finta semplice (Salzburg 1769) von Wolfgang Amadeus Mozart gemein, dessen Libretto von Marco Coltellini bekanntlich auf das Libretto von Carlo Goldoni für das gleichnamige Dramma giocoso von Salvatore Perillo zurückgeht (Venedig 1764).

32. «La finta semplice o sia Il tutore burlato. Dramma giocoso da rappresentarsi in Lisbona nel Teatro della Rua dos Condes nella primavera dell'anno 1773. Nella Stamperia Reale.»

33. Vgl. Arturo Zabala, La opera en la vida teatral valenciana del siglo XVIII, Valencia 1960, S. $147 \mathrm{u} .261 \mathrm{ff}$., sowie Francisco Aguilar PIÑal, Sevilla y el teatro en el siglo XVIII, S. 131 u. 284.

34. Das Werk wurde 1782 von der Compañía von Eusebio Ribera in italienischer Sprache aufgeführt. Vgl. Cotarelo y Mori, Orígenes y establecimiento de la ópera en España hasta 1800, S. 291.

[8] 
den unmittelbaren Vergleich mit Paisiellos ,Erfolgsoper'vermieiden wollte und dennoch diesen Stoff als ausgesprochen attraktiv empfand. Ob diese Wahl jedoch auf ihn oder einen Auftraggeber — den Hof oder einen Impresario- zurückging und ob ihm das Lissabonner Textbuch aus Neapel oder unmittelbar aus Lissabon bekannt war, ist unklar.

Aus der Tatsache, daß Martín y Soler in gedruckten Libretti der 1780er Jahre als in Diensten des Príncipe de Asturias, also des spanischen Thronfolgers, des zukünftigen Carlos IV., bezeichnet ist, wurde oft auf eine Verbindung zu diesem noch vor seinem neapolitanischen Aufenthalt geschlossen ${ }^{35}$. Tatsächlich ist Martín y Soler erst 1780 im Libretto zu L'Ipermestra als «maestro di capp. spagnolo al serv. del princ. d'Asturias» ${ }^{36}$ bezeichnet, während ihn das Libretto der im Jahr zuvor aufgeführten Ifigenia in Aulide noch lediglich als «maestro di capp. spagnolo» angibt ${ }^{37}$.

Zwar finden sich in den Akten des Palastarchivs von Madrid keinerlei Hinweise auf irgendeine Anstellung Martín y Solers am spanischen Hof, doch belegen die Rechnungsbücher des «Cuarto del Príncipe» immerhin konkrete Kontakte zwischen Martín y Soler und dem Príncipe de Asturias. Während für 1778 im Haushalt der Prinzen - wie auch in dem des Königs - noch keinerlei Ausgaben für Martín y Soler verzeichnet sind ${ }^{38}$, wird 1780 eine Ausgabe für 1779 erwähnt, die nahelegt, daß Martín y Soler sich in diesem Jahr (wieder) am spanischen Hof aufgehalten hat. Unter den Gratificaciones für 1780 ist aufgeführt:

«A D. ${ }^{\mathrm{n}}$ Vicente Pietrasanta, primer Teniente de la Compañia Italiana de R. ${ }^{\mathrm{s}}$ Guardias de Corps se le entregaron \#dos mil seiscientos diez $\mathrm{r}^{\mathrm{s}}{ }^{\text {vell. }}{ }^{\mathrm{n}}$ [reales de vellón] que $\mathrm{S}$. A. se dignò conceder à D. "Vicente Martín, Musico en Napoles, por su asistencia à la diversion de Parejas en el año proximo pasado de 1779 , segun Libram. ${ }^{\text {to }}$ de trece de septiembre $n .^{\circ} 17 .{ }^{39}$

Da Martín y Soler sich im Januar 1779 zur Aufführung seiner Ifigenia in Aulide zweifellos (wieder) in Neapel befand, wird man davon ausgehen müssen, daß er im Frühjahr oder Sommer dieses Jahres wieder nach Spanien reiste, wo er dann aller Wahrscheinlichkeit nach in einem der königlichen Schlösser an der erwähnten «diversion de Parejas» mitwirkte (vermutlich mit eigenen Kompositionen). Offensichtlich intensivierten sich bei dieser Gelegenheit die Kontakte zwischen Martín y Soler und dem spanischen Thronfolger, denn Anfang 1780 ver-

35. Vgl. den Artikel "Martín y Soler" von Dorothea Link in The New Grove Dictionary of Opera, Bd. 3, S. 240: "Born and raised in Valencia, he entered the service of the Prince of Asturias, the future Charles IV of Spain, in about 1776, the year in which his first opera was probably given in Madrid."

36. SARTORI, I libretti italiani a stampa, Nr. 13602.

37. Ebda., Nr. 12729.

38. Dies hängt wohl nicht zuletzt damit zusammen, daß der Kronprinz sich in diesem Jahr nicht in San Ildefonso aufhielt, sondern wegen einer Erkrankung seiner Gemahlin in El Escorial geblieben war.

39. Archivo del Palacio Real de Madrid, Carlos IV (Príncipe), Leg. 8, Cuentas del Cuarto del Príncipe 1779-81. 
zeichnen die Rechnungsbücher die beachtliche Summe von 10950 Reales de vellón für Kopien, die Martín y Soler diesem überreicht oder zugesandt hatte:

«A D. ${ }^{n}$ Vicente Martín, Maestro de Capilla en Napoles, se le pagaron por mano del Mariscal de Campo D. ${ }^{n}$ Vicente Pietrasanta por varias copias de Musica, que ha remitido à S. A. como consta del harebueno su fecha veinte y dos de Febrero n..$^{\circ} 3$..... 10950».

Unter den Kopien dürfte sich auch die kurz zuvor in Neapel aufgeführte Ifigenia in Aulide befunden haben, da die Biblioteca del Palacio Real de Madrid eine handschriftliche Partitur dieses Werks aufbewahrt. Schließlich findet sich im darauffolgenden Jahr auch ein Beleg für die Übersendung einer Kopie der Andromaca, die am 26. Dezember 1780 in Turin uraufgeführt worden war: Am 8. Mai 1781 wurden 640 Reales de vellón einem Dom.[enico] Marcoleta angewiesen, "por cuya disposicion se pagaron en Turín à D. ${ }^{n}$ Vizente Martín, por la copía en musica de una opera titulada la Andromaca, que remitió à S. A.»

Wie die wenigen überlieferten Dokumente erkennen lassen, geht die Tatsache, daß Martín y Soler sich ab 1780 als in Diensten des Príncipe de Asturias stehend bezeichnete, also entgegen bisheriger Auffassung nicht unmittelbar auf Kontakte zum spanischen Hof vor seinem Aufenthalt in Neapel zurück, sondern dürfte eine Folge des Aufenthalts am spanischen Hof von 1779 gewesen $\operatorname{sein}^{40}$. Dabei muß einstweilen offen bleiben, ob es einen Madrider Aufenthalt vor 1779 überhaupt gab, ob seine Tätigkeit in Neapel vielleicht vom Madrider Hof protegiert wurde oder ob nicht sogar umgekehrt die Kontakte zum spanischen Hof auf Einfluß aus Neapel zurückzuführen sind.

Dies alles sind nur einige Mosaiksteine, die letztlich mehr Fragen aufwerfen, als sie beantworten. Diese Fragen betreffen jedoch nicht nur die Biographie Martín y Solers, sondern zielen darüber hinaus auf grundsätzliche Probleme der Geschichte des spanischen Musiktheaters im 18. Jahrhundert. So ist beispielsweise die Musiktheaterpraxis an den Reales Sitios außerhalb Madrids bisher kaum untersucht worden ${ }^{41}$. Auch ist die häufig geäußerte Ansicht, daß dort nach 1777 keine Opernaufführungen mehr stattfanden, allem Anschein nach irrig. Was sich geändert haben dürfte, sind die Bedingungen, unter denen Opern produziert wurden. Dabei läuft eine fast ausschließlich an gedruckten Libretti orientierte positivistische Historiographie, wie sie in Spanien von Emilio Cotarelo y Mori geprägt wurde ${ }^{42}$, notwendigerweise Gefahr, Phänomene,

40. Dabei kam allerdings allem Anschein nach nie ein Dienstverhältnis im engeren Sinne zustande, denn in den Gehaltslisten ist Martín y Soler nie aufgeführt, auch nicht unter den «Sueldos extraordinarios», obwohl im Haushalt des Kronprinzen mehrere Musiker, darunter der Violinist Cayetano Bruneti [Brunetti] angestellt waren.

41. Sartori verzeichnet die folgenden Libretti zu Opernaufführungen an den Reales Sitios außerhalb Madrids: L'impresario delle Canarie (Intermezzi, Metastasio/Leo?), Il Pardo 1739; Il tuttore o sia La pupilla (Intermezzi, ?/Hasse), Real Sito del Pardo 1740; La festa cinese (Componimento drammatico, Metastasio/Conforto), Regio palagio del Real Sito d'Aranjuez 1751; L'isola disabitata (Azione per musica, Metastasio/Bonno), Aranjuez 1753; La fedeltà premiata (Pastorale), Teatri delli Reali Siti 1766; La buona figliuola maritata (Dramma giocoso, Goldoni/Piccinni), Teatro di San Ildefonso Sommer 1769; Le gemelle (Tonioli/Tozzi), Real Sito di S. Ildefonso 1776. Vgl. in diesem Zusammenhang auch den Aufsatz von José Domingo Delgado Bedmar, «El «Coliseo de Comedias» de San Ildefonso en el siglo XVIII», in: El Arte en las Cortes Europeas del siglo XVIII, Comunicaciones del Congreso Madrid y Aranjuez, 27-29 abril 1987, Madrid, Comunidad de Madrid, 1989, S. $229 \mathrm{ff}$.

42. Vgl. die Einleitung («Introducción. Problemas, métodos y perspectivas») des Verfassers in Teatro y Música en España (siglo XVIII), Actas del Simposio Internacional Salamanca 1994, hrsg. von R. Kleinertz, Kassel und Berlin, Edition Reichenberger, 1996. 
die so nicht dokumentiert sind, aus dem Blick zu verlieren. So ist es kein Zufall, daß in der überaus materialreichen Arbeit Cotarelos über die Orígenes y establecimiento de la ópera en España hasta 1800 (Madrid 1917) das Jahr 1778 schlicht übergangen wird. Die Existenz einer Partitur Martín y Solers, die auf die Aufführung einer italienischen Oper in San Ildefonso im Jahre 1778 hinweist, mahnt hier einmal mehr zur Vorsicht. 